\title{
Information Prescriptions: The Key to Unlocking Behaviour Change
}

Authors: Robin Hewings (Diabetes UK), Mhukti Perumal (Diabetes UK), Amy Rylance (Diabetes UK), Rezaul Mirza (Diabetes UK)

\section{Background}

The National Diabetes Audit 2015-2016 highlights the challenge of self-care for the 4.5 million people in the UK with diabetes. Only $40.2 \%$ of people with Type 2 diabetes achieve the National Institute of Health and Care Excellence (NICE) recommended targets for HbA1c, blood pressure and cholesterol. Failure to meet these targets can lead to devastating and life-threatening complications, including heart disease, amputation and stroke.

Diabetes UK has created Information Prescriptions (IPs). The short, easy to read and clinically accurate resources help healthcare professionals and people with diabetes to make decisions together about the treatment and self-management of their diabetes.

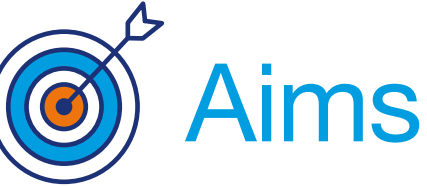

To develop personalised IPs that:

- Give people with diabetes the information they need to understand and engage with their diabetes.

- Give healthcare professionals (particularly in primary care) a tool they can easily use to support care planning and motivate patients to change their behaviour.

\section{Method}

\section{Getting the information right}

We ran two co-production workshops with clinicians, health academics and people with diabetes to ensure that we included the right information and used the right language.

\section{Testing the tool}

We piloted IPs at three surgeries. This was to test if healthcare professionals found the tool easy to use and to see if patients understood and engaged with the information given.

3 Integrating the information into clinical practice To make the tool easily accessible, we worked with the three main primary care IT providers in the UK to integrate the information into clinical practice. This enabled us to:

- Target those at highest risk. A pop-up alert, to fill in an IP, opens on the patient's medical record if they fall out outside of NICE recommended targets for $\mathrm{HbA} 1 \mathrm{c}$, blood pressure or cholesterol.

- Support care planning. Simple check boxes allow generic advice to be individualised whilst the 'Action Plan' box draws on the best evidence around effective behaviour change.

- Enables continuity of care. The completed IP is saved in the patient's notes and can be recalled in future appointments to enable quick recall of past goals.

\section{Results}

\section{Changing clinical behaviour}

Case studies show the IPs are prompting clinicians to intervene more proactively and identify patients who have slipped through the net for many years.

Data from clinical system EMIS Web shows that use of IPs is rising steadily and they have now been used in over 110,000 clinical consultations.

\section{Increasing patient} understanding of key concepts Many patients say the IP was the first time anyone explained their diabetes to them.

\section{Prompting patient action}

We are hearing of patients significantly improving clinical outcomes (see case study below). The IPs include a unique link to more information on Diabetes UK's website. Over 35,000 patients were successfully motivated by the receipt of an IP to access more information on managing their diabetes.
"They are hands down the most useful patient resource I have encountered in my diabetes career to date." Dr Kevin Fernando, GP

"Well for me it was the integrated prompt in IT, by far that was the most useful thing... That's how I first became aware of them, and that's why I keep remembering about them." Transcripts from Newcastle University evaluation

"Individuals report a clearer understanding of the concepts and relevance of $\mathrm{HbA} 1 \mathrm{c}$, lipids and blood pressure and an enhanced motivation to adopt lifestyle modification specific to their own needs." Nicola Milne, practice nurse

\section{fôn Case study}

The Waverley Medical Practice in Scotland did an audit of the IPs. They showed that after the introduction of the IPs, there was a 10 per cent increase in patients hitting $\mathrm{HbA} 1 \mathrm{c}$ targets.

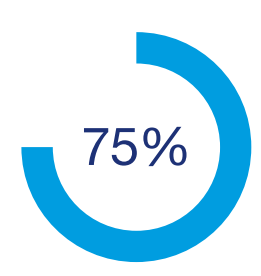

T2DM patients with $\mathrm{HbA1c}<58$ at Baseline

\section{$85 \%$}

\section{T2DM Patients} with $\mathrm{HbA1c}<58$ one year later
The IPs won runner up in 2016 at the British Medical Association's Patient Information Awards and won a Quality in Care for Diabetes Award in 2016.

\section{Riscussion}

IPs are reaching the people at highest risk of diabetes complications with an evidence-based intervention to help them understand and take ownership of their care. From the results shown we can see that personal and targeted IPs should become a key tool for clinicians delivering quality diabetes care.

"The information prescriptions help me to plan better if there is a problem with my test readings." Person with diabetes 